\title{
Spatio-temporal structure of Alfvén waves excited by a sudden impulse localized on an L-shell
}

\author{
D. Yu. Klimushkin ${ }^{1}$, P. N. Mager ${ }^{1}$, and K.-H. Glassmeier ${ }^{2}$ \\ ${ }^{1}$ The Institute of Solar-Terrestrial Physics of Siberian Branch of Russian Academy of Sciences, P.O. Box 291, \\ 664033 Irkutsk, Russia \\ ${ }^{2}$ Institut für Geophysik und Extraterrestrische Physik, Mendelssohnstrasse 3, 38106 Braunschweig, Germany \\ Correspondence to: D. Yu. Klimushkin (klimush@iszf.irk.ru)
}

Received: 27 March 2012 - Revised: 25 June 2012 - Accepted: 3 July 2012 - Published: 25 July 2012

\begin{abstract}
This paper is concerned with the spatial structure and temporal evolution of the azimuthally small scale Alfvén wave generated by a sudden impulse concentrated on a given magnetic shell. At the outset, both poloidal and toroidal components are present in the wave's magnetic field. The oscillation in the poloidal component on a given magnetic shell is a superposition of two monochromatic oscillations, one with the local resonance frequency on this shell, and the other with the frequency corresponding to the resonance frequency on the source surface. The superposition of these two oscillations leads to beating. Due to phase mixing, the poloidal component of the oscillation decreases with time down to zero, transferring its energy to the toroidal component. Beating in the toroidal component is less pronounced. As time elapses, energy concentration near the source magnetic shell occurs with the frequency of the oscillation corresponding to the Alfvénic resonance frequency on this surface. Outside this thin region wave amplitudes become rather small at oscillation frequencies corresponding to the local resonance frequency of the respective magnetic shell.
\end{abstract}

Keywords. Magnetospheric physics (MHD waves and instabilities)

\section{Introduction}

A general framework for the interpretation of ultra-low frequency (ULF) wave phenomena in planetary magnetospheres is the concept of the field line resonance: a fast magnetosonic wave is generated on the magnetopause and propagates into the inhomogeneous magnetosphere generating an Alfvén mode on a surface where the wave frequency equals the local Alfvén frequency (for a more comprehensive overview see Glassmeier et al., 1999). As a result, the toroidal Pc5 Alfvén wave is observed in the magnetosphere and on the ground (e.g. Agapitov et al., 2009). This process is effective only in the case of the low- $m$ initial perturbation (where $m$ is the azimuthal wave number), since in the opposite case the magnetosphere is opaque for the fast magnetosonic wave. The initial fast mode can be generated by a process on the surface of the magnetosphere, e.g. by Kelvin-Helmholtz or Kruskal-Schwarzschild instability (e.g. Mishin, 1993; Fujita et al., 1996; Plaschke and Glassmeier, 2011; Mazur and Chuiko, 2011).

Surface perturbations on the magnetopause are also supposed to generate traveling convection vortices in the ionosphere (Kivelson and Southwood, 1991; Glassmeier, 1992; Chi et al., 2006). In this case the perturbation is highly localized in the azimuthal direction, which means that it mainly consists of the high- $m$ harmonics. Therefore, this kind of perturbations must have Alfvénic character, being mostly the parallel current disturbances (Glassmeier, 1992). Nevertheless, a compressional magnetic field perturbation can appear due to finite plasma pressure and field line curvature effects (e.g. Mager and Klimushkin, 2002).

This picture was initially established for a monochromatic driving wave, which is not a realistic case, since all wave phenomena in the magnetosphere are related to finite-duration processes. Several theoretical studies (Chen and Hasegawa, 1974; Radoski, 1974; Mann and Wright, 1995; Leonovich and Mazur, 1998; Klimushkin and Mager, 2004; Klimushkin et al., 2007) addressed the problem of the spatio-temporal evolution of such an initial finite-duration disturbance across magnetic shells. It was shown that in the course of the 
evolution the wave field structure is governed by a phenomenon known as phase mixing. At the initial onset of the perturbation, all field lines oscillate with the same phase and the wave field is characterized by a predominantly poloidal polarization. However, since each field line oscillates with its own eigenfrequency, the oscillations on neighboring magnetic shells rapidly acquire significant phase differences. As a consequence, the wave acquires a very small spatial scale across magnetic field shells and, hence, becomes toroidally polarized to preserve the source-free nature of the magnetic field.

However, since the initial disturbance is often viewed as a surface wave on the magnetopause, it is of interest to consider a case when the Alfvén wave is excited by an impulsive source concentrated on a specific magnetic shell. For this special case, Hasegawa et al. (1983) found features similar to those of the case with a source widely distributed across L-shells. In our present study, we reexamine the problem as the solution obtained by Hasegawa et al. (1983) does not take into account surface wave features.

In Sect. 2 we formulate an equation describing the spatiotemporal structure of a high- $m$ wave generated by a sudden impulse concentrated on a given magnetic shell. In Sect. 3 we perform a Fourier-transform of the equation over time obtaining as a result an integral solution for a spatial structure of a harmonic with a definite frequency. In Sect. 4 we perform an inverse Fourier-transform, that is, we integrate the obtained monochromatic solution over frequency. In Sect. 5 we apply a saddle-point method to the integral found in the previous section which allows us to find an asymptotic solution of our problem. In Sect. 6 we discuss some consequences of our solution. Section 7 presents the principal results of the paper.

\section{Initial equations}

In this study the magnetosphere is approximated and represented as a rectangular box. A field-aligned Cartesian coordinate system is used: the $\mathrm{z}$-coordinate is directed along the field lines, the $\mathrm{x}$-coordinate is directed across the magnetic field lines and takes the role of the radial coordinate in the magnetosphere, and the y-coordinate is directed both across field lines and magnetic shells, completing the triad and taking the role of the azimuthal coordinate. Plasma parameters are assumed to vary only in the x-direction; thus, all perturbed quantities on the azimuthal and longitudinal coordinates vary as $e^{i k_{\mathrm{y}} y+i k_{\mathrm{z}} z}$.

The equilibrium values of the magnetic field and plasma density are designated as $\boldsymbol{B}$ and $\rho$, respectively; $\boldsymbol{\xi}$ denotes the displacement of plasma from its equilibrium position, $\boldsymbol{E}$, $\boldsymbol{b}$ and $\boldsymbol{j}$ are the electric field, magnetic field, and electric current density of the wave, respectively.

The wave is assumed to be excited by a sudden impulse of an azimuthal current density $\boldsymbol{J}=\left(0, J_{\mathrm{y}}, 0\right)$ with

$J_{\mathrm{y}}=J_{0} \delta\left(x-x_{0}\right) \delta\left(t-t_{0}\right)$ with $t_{0}$ denoting the onset time at the surface with radial coordinate $x_{0}$. Following Akhiezer et al. (1975), this driving external current $\boldsymbol{J}$ should be considered as an additional term in Ampere's law:

$\nabla \times \boldsymbol{b}=\frac{4 \pi}{c} \boldsymbol{j}+\frac{4 \pi}{c} \boldsymbol{J}$.

The linearized equation of motion for small oscillations takes the form

$\rho \frac{\partial^{2} \boldsymbol{\xi}}{\partial t^{2}}-\frac{1}{c} \boldsymbol{j} \times \boldsymbol{B}=0$

where

$\nabla \times \boldsymbol{E}=-\frac{1}{c} \frac{\partial \boldsymbol{b}}{\partial t}$

and

$\boldsymbol{E}=\frac{1}{c} \frac{\partial \boldsymbol{\xi}}{\partial t} \times \boldsymbol{B}$

Using these equations, one obtains the equation for the wave electric field $\boldsymbol{E}(x, t)$ :

$\frac{1}{A^{2}} \frac{\partial^{2} \boldsymbol{E}}{\partial t^{2}}-\nabla \times \nabla \times \boldsymbol{E}=-\frac{4 \pi}{c^{2}} \frac{\partial \boldsymbol{J}}{\partial t}$,

where $A=B / \sqrt{4 \pi \rho}$ is the Alfvén speed.

Let us suppose that the region under investigation lies in the opaque region for the fast mode. For example, it is possible when a high- $m$ perturbation is considered. Then the only propagating wave mode is the Alfvén mode. Assuming an infinite plasma conductivity in the magnetic field direction, the parallel electric field component may be neglected; thus, the wave's electric field lies on surfaces orthogonal to the field lines. According to the Helmholtz theorem (Morse and Feshbach, 1953), an arbitrary vector field can split into the sum of potential and vortical components. By applying this theorem to the two-dimensional field $\boldsymbol{E}$, we use the representation $\boldsymbol{E}=-\nabla_{\perp} \Phi+\nabla_{\perp} \times \boldsymbol{e}_{\|} \Psi$, where $\boldsymbol{e}_{\|}=\boldsymbol{B} / B$ and $\nabla_{\perp}$ is the transverse nabla-operator. The "potentials" $\Phi$ and $\Psi$ represent the electric field of the Alfvén wave and fast mode, respectively (Klimushkin, 1994; Fedorov et al., 1998). As we are considering a region lying deep within the fast mode opaque region, the fast mode contribution $\Psi$ can be neglected, and the electric field of the wave can be represented in the form

$\boldsymbol{E}=-\nabla_{\perp} \Phi$.

Let us substitute Eq. (7) into Eq. (6) and apply the operator $\nabla_{\perp}$ on the expression obtained by this substitution. As a result, we find the equation

$$
\begin{array}{r}
\frac{\partial}{\partial x}\left[-\frac{1}{A^{2}} \frac{\partial^{2}}{\partial t^{2}}-k_{\mathrm{z}}^{2}\right] \frac{\partial \Phi}{\partial x}-k_{\mathrm{y}}^{2}\left[-\frac{1}{A^{2}} \frac{\partial^{2}}{\partial t^{2}}-k_{\mathrm{z}}^{2}\right] \Phi \\
=-\frac{4 \pi i k_{\mathrm{y}}}{c^{2}} \frac{\partial J_{\mathrm{y}}}{\partial t} .
\end{array}
$$




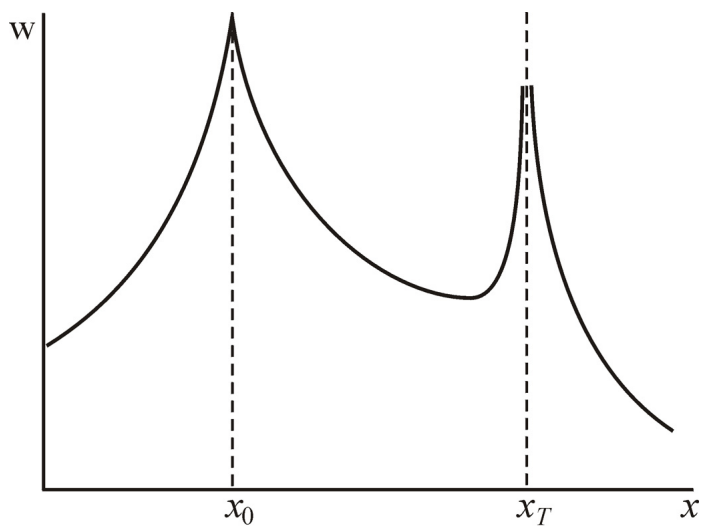

Fig. 1. A surface wave with superposed field line resonance. Here $w$ is the wave energy density.

As a boundary condition for the solution of this partial differential equation we use the condition

$\left.\Phi\right|_{x \rightarrow \pm \infty}=0$.

\section{Solution for a single Fourier-harmonic}

Performing a Fourier-transform over time, we have

$$
\begin{aligned}
& \frac{\partial}{\partial x}\left[\frac{\omega^{2}}{A^{2}}-k_{\mathrm{z}}^{2}\right] \frac{\partial \Phi_{\omega}}{\partial x}-k_{\mathrm{y}}^{2}\left[\frac{\omega^{2}}{A^{2}}-k_{\mathrm{z}}^{2}\right] \Phi_{\omega} \\
& =q \delta\left(x-x_{0}\right) \omega e^{i \omega t_{0}},
\end{aligned}
$$

where $\Phi_{\omega}$ is a Fourier-transformed $\Phi(t)$ value, and

$q=-4 \pi J_{0} k_{\mathrm{y}} / c$.

This is a differential equation describing a monochromatic Alfvén oscillation generated by the external current in the approximation when the fast mode can be neglected. The same equation can be obtained as an exact one by assuming the plasma to be an incompressible fluid (Hasegawa and Uberoi, 1982).

Let us introduce the resonance (toroidal) eigenfrequency as $\Omega(x)=k_{\mathrm{Z}} A(x)$. Then the resonance surface $x_{T}$ can be determined as a surface where the equality $\omega=\Omega(x)$ holds. Assuming the linear profile for the resonance frequency,

$\Omega=\Omega_{0} \cdot \frac{l-x}{l}$,

the coordinate of the resonance surface is

$x_{T}(\omega)=l \cdot \frac{\Omega_{0}-\omega}{\Omega_{0}}$.

Near this surface, an approximate equality $\omega^{2}-\Omega^{2} \approx$ $2 \omega(\omega-\Omega)$ holds, and Eq. (10) can be rewritten in the form

$$
\begin{array}{r}
\frac{\partial}{\partial x}\left[x-x_{T}(\omega)\right] \frac{\partial \Phi_{\omega}}{\partial x}-k_{\mathrm{y}}^{2}\left[x-x_{T}(\omega)\right] \Phi_{\omega} \\
=q \frac{l}{2 \Omega_{0}} e^{i \omega t_{0}} \delta\left(x-x_{0}\right) .
\end{array}
$$

The solution of this equation can be written in the integral form

$\Phi_{\omega}=q \frac{l}{2 \Omega_{0}} e^{i \omega t_{0}} \int_{-\infty}^{+\infty} d k \frac{e^{i k\left(x-x_{T}\right)}}{\sqrt{k_{\mathrm{y}}^{2}+k^{2}}} \int_{-\infty}^{k} d k^{\prime} \frac{e^{i k^{\prime}\left(x_{T}-x_{0}\right)}}{\sqrt{k_{\mathrm{y}}^{2}+k^{\prime 2}}}$

(Leonovich and Mazur, 1999). This expression describes a surface wave exponentially decreasing from the surface of the source $x_{0}$ with a field line resonance superposed on this background (Fig. 1). It should be noticed that it is a different kind surface wave than considered by, e.g. Hasegawa and Chen (1974), DeKeyser et al. (1999), or Plaschke and Glassmeier (2011), since it is not related to a sharp inhomogeneity in the medium where wave propagation is considered; however, this surface wave is due to the highly localized source described by the azimuthal current density $\boldsymbol{J}=\left(0, J_{\mathrm{y}}, 0\right)$. In cases where perturbations are caused by magnetospheric buffeting (e.g. Kivelson and Southwood, 1991; Glassmeier, 1992), the localized source would be located right at the magnetopause.

\section{Inverse Fourier-transform over time}

Performing the inverse Fourier-transform of Eq. (12) over time, we find a solution in the form

$$
\begin{aligned}
\Phi(x, t)= & \frac{l q}{2 \Omega_{0}} \int_{-\infty}^{\infty} d \omega e^{-i \omega\left(t-t_{0}\right)} \int_{-\infty}^{\infty} d k \frac{e^{i k\left[x-x_{T}(\omega)\right]}}{\sqrt{k_{\mathrm{y}}^{2}+k^{2}}} \\
& \cdot \int_{-\infty}^{k} d k^{\prime} \frac{e^{\left.i k^{\prime}\left[x_{T}(\omega)-x_{0}\right)\right]}}{\sqrt{k_{\mathrm{y}}^{2}+k^{\prime 2}}} .
\end{aligned}
$$

Taking into account the linear $x_{T}(\omega)$ dependence of Eq. (11), this expression can be rewritten as

$$
\begin{aligned}
\Phi= & \frac{l q}{2 \Omega_{0}} \int_{-\infty}^{\infty} d k \frac{e^{i k(x-l)}}{\sqrt{k_{\mathrm{y}}^{2}+k^{2}}} \int_{-\infty}^{k} d k^{\prime} \frac{e^{-i k^{\prime}\left(x_{0}-l\right)}}{\sqrt{k_{\mathrm{y}}^{2}+k^{\prime 2}}} \\
& \cdot \int_{-\infty}^{\infty} d \omega e^{-i \omega\left[\left(t-t_{0}\right)-\frac{l}{\Omega_{0}}\left(k-k^{\prime}\right)\right]} .
\end{aligned}
$$

Noting that the integral over $\omega$ here is an integral representation of the delta-function,

$$
\int_{-\infty}^{\infty} d \omega e^{-i \omega\left[\left(t-t_{0}\right)-\frac{l}{\Omega_{0}}\left(k-k^{\prime}\right)\right]}=2 \pi \delta\left[\left(t-t_{0}\right)-\frac{l}{\Omega_{0}}\left(k-k^{\prime}\right)\right],
$$

the integration over $k^{\prime}$ can be easily performed. Finally, we obtain the following solution:

$$
\Phi(x, t)=q \pi \Theta\left(t-t_{0}\right) e^{i\left(t-t_{0}\right) \frac{\Omega_{0}}{T}\left(x_{0}-l\right)} I,
$$


where

$I=\int_{-\infty}^{\infty} d k \frac{e^{i k(x-l)-i k\left(x_{0}-l\right)}}{\sqrt{k_{\mathrm{y}}^{2}+k^{2}} \sqrt{k_{\mathrm{y}}^{2}+\left[k-\frac{\Omega_{0}}{l}\left(t-t_{0}\right)\right]^{2}}}$.

Thus, only the integration over $k$ has yet to be performed.

\section{Asymptotic solution}

Let us represent the integral in Eq. (14) in the form

$I=\int_{-\infty}^{\infty} d k e^{S(k)}$

where the "phase" is

$S=i k\left(x-x_{0}\right)-\frac{1}{2} \ln \left(k_{\mathrm{y}}^{2}+k^{2}\right)-\frac{1}{2} \ln \left[k_{\mathrm{y}}^{2}+\left(k-\frac{\Omega_{0}}{l}\left(t-t_{0}\right)\right)^{2}\right]$.

To find an asymptotic solution at $k_{\mathrm{y}}\left|x_{0}-x\right| \gg 1$, we can use the saddle-point method. The saddle-point is determined as a point where the derivative $d S / d k$ vanishes:

$\frac{d S}{d k}=i\left(x-x_{0}\right)-\frac{k}{k_{\mathrm{y}}^{2}+k^{2}}-\frac{k-\frac{\Omega_{0}}{l}\left(t-t_{0}\right)}{k_{\mathrm{y}}^{2}+\left[k-\frac{\Omega_{0}}{l}\left(t-t_{0}\right)\right]^{2}}=0$.

A large value of $k_{\mathrm{y}}\left|x-x_{0}\right|$ implies that the denominator of either the second or the third term is small. That is, there are four saddle-points,

$k_{1} \approx+i k_{\mathrm{y}}$

$k_{2} \approx-i k_{\mathrm{y}}$

$k_{3} \approx+i k_{\mathrm{y}}+\frac{\Omega_{0}}{l}\left(t-t_{0}\right)$

$k_{4} \approx-i k_{\mathrm{y}}+\frac{\Omega_{0}}{l}\left(t-t_{0}\right)$

If we are searching the solution in the region $x<x_{0}$, then the path of the integration should go though the points $k_{2}$ and $k_{4}$. Correcting for a finite $k_{\mathrm{y}}\left|x-x_{0}\right|$ value yields

$k_{2}=-i k_{\mathrm{y}}+\frac{i}{2\left(x_{0}-x\right)}$

and

$k_{4}=-i k_{\mathrm{y}}+\frac{\Omega_{0}}{l}\left(t-t_{0}\right)+\frac{i}{2\left(x_{0}-x\right)}$.

In the vicinity of these two points, the "phase" can be approximated as

$S \approx S\left(k_{2,4}\right)+\frac{1}{2} \frac{d^{2} S\left(k_{2,4}\right)}{d k^{2}}\left(k-k_{2,4}\right)^{2}$ where

$$
\begin{aligned}
S\left(k_{2}\right) & =-k_{\mathrm{y}}\left(x_{0}-x\right)-\ln \sqrt{\frac{k_{\mathrm{y}}}{x_{0}-x}} \\
& -\ln \sqrt{\frac{\Omega_{0}^{2}}{l^{2}}\left(t-t_{0}\right)^{2}+2 i k_{\mathrm{y}} \frac{\Omega_{0}}{l}\left(t-t_{0}\right),}
\end{aligned}
$$

$$
\begin{aligned}
S\left(k_{4}\right) & =-k_{\mathrm{y}}\left(x_{0}-x\right)+i \frac{\Omega_{0}}{l}\left(t-t_{0}\right)\left(x-x_{0}\right)-\ln \sqrt{\frac{k_{\mathrm{y}}}{x_{0}-x}} \\
& -\ln \sqrt{\frac{\Omega_{0}^{2}}{l^{2}}\left(t-t_{0}\right)^{2}-2 i k_{\mathrm{y}} \frac{\Omega_{0}}{l}\left(t-t_{0}\right)},
\end{aligned}
$$

and

$\frac{d^{2} S\left(k_{2}\right)}{d k^{2}}=\frac{d^{2} S\left(k_{4}\right)}{d k^{2}}=-2\left(x-x_{0}\right)^{2}$.

Now the integral $I$ can be approximately represented as a sum of two integrals near the two saddle-points,

$$
I=I_{2}+I_{4}
$$

with

$$
\begin{aligned}
I_{2,4} & =\int_{-\infty}^{\infty} d\left(k-k_{2,4}\right) e^{S\left(k_{2,4}\right)-\left(x-x_{0}\right)^{2}\left(k-k_{2,4}\right)^{2}} \\
& =\frac{\sqrt{\pi}}{\sqrt{k_{\mathrm{y}}\left(x_{0}-x\right)}} \frac{e^{-k_{\mathrm{y}}\left(x_{0}-x\right)}}{\sqrt{\frac{\Omega_{0}^{2}}{l^{2}}\left(t-t_{0}\right)^{2} \pm 2 i k_{\mathrm{y}} \frac{\Omega_{0}}{l}\left(t-t_{0}\right)}} .
\end{aligned}
$$

Finally, we have for the wave electric field potential

$$
\Phi(x, t)=q \pi^{3 / 2} \Theta\left(t-t_{0}\right) \frac{e^{-k_{\mathrm{y}}\left(x_{0}-x\right)}}{\sqrt{k_{\mathrm{y}}\left(x_{0}-x\right)}}\left[F_{\mathrm{X}}(t)+F_{0}(t)\right],
$$

where

$$
\begin{aligned}
& F_{\mathrm{x}}(t)=\frac{e^{-i \Omega(x)\left(t-t_{0}\right)}}{\sqrt{\frac{\Omega_{0}^{2}}{l^{2}}\left(t-t_{0}\right)^{2}-2 i k_{\mathrm{y}} \frac{\Omega_{0}}{l}\left(t-t_{0}\right)}}, \\
& F_{0}(t)=\frac{e^{-i \Omega\left(x_{0}\right)\left(t-t_{0}\right)}}{\sqrt{\frac{\Omega_{0}^{2}}{l^{2}}\left(t-t_{0}\right)^{2}+2 i k_{\mathrm{y}} \frac{\Omega_{0}}{l}\left(t-t_{0}\right)}},
\end{aligned}
$$

and $\Omega(x)$ and $\Omega\left(x_{0}\right)$ are the resonance frequencies on the given magnetic shell $(x)$ and on the magnetic shell of the source $\left(x_{0}\right)$, respectively. 


\section{Analysis of the solution}

The factor $\exp \left[-k_{\mathrm{y}}\left(x_{0}-x\right)\right]$ in Eq. (15) shows that the solution possesses the features of a surface wave localized on the magnetic shell of the source $x_{0}$. On this "background" surface wave an additional spatial oscillation is imposed described by the factor $\exp \left[-i \Omega(x)\left(t-t_{0}\right)\right]$ in the term $F_{\mathrm{X}}(t)$. This spatial modulation is displayed in Fig. 2, representing the result of a numerical determination of the integral (14).

The characteristic radial wave vector can be determined as $k_{\mathrm{x}}=\frac{\partial \Omega(x)}{\partial x}\left(t-t_{0}\right)$.

The increase of the radial wave vector with time (which can also be inferred from Fig. 2) implies a transformation from a predominantly poloidal Alfvén mode (large $E_{\mathrm{y}}$ component) into a toroidally polarized Alfvén mode (large $E_{\mathrm{x}}$ component) since the wave the electric fields components are determined by $E_{\mathrm{y}}=-i k_{\mathrm{y}} \Phi$ and $E_{\mathrm{x}}=-\partial \Phi / \partial x$. The decrease of the radial wave length as well as the polarization change is due to the phase mixing and is required to keep the magnetic field perturbation source-free.

Let us consider the temporal evolution of the wave field in more detail. For this we calculate the azimuthal electric field of the wave:

$$
E_{\mathrm{y}}=-i k_{\mathrm{y}} q \pi^{3 / 2} \Theta\left(t-t_{0}\right) \frac{e^{-k_{\mathrm{y}}\left(x_{0}-x\right)}}{\sqrt{k_{\mathrm{y}}\left(x_{0}-x\right)}}\left[F_{\mathrm{x}}(t)+F_{0}(t)\right] .
$$

The first term in this expression, $F_{\mathrm{X}}(t)$, corresponds to the oscillation with the local resonance frequency, as in the theory of the wave excited by the impulse widely distributed across magnetic shells (below, this theory is referred to as the wide impulse theory). However, there is the second term, $F_{0}(t)$, describing the oscillation with a frequency corresponding to the resonance frequency on the source surface, which does not have an analogy in the wide impulse theory. As both, the local resonance frequency and the resonance frequency of the source surface are different, beating occurs as seen in Fig. 3. Due to the presence of the square roots in the denominators of $F_{\mathrm{X}}(t)$ and $F_{0}(t)$, the amplitude of the oscillation in the $E_{\mathrm{y}}$ component decreases with time, which is also evident from Fig. 4, where the wave electric field energy density $w_{\mathrm{y}}=\left|E_{\mathrm{y}}\right|^{2} / 8 \pi$ is displayed as a function of time. The poloidal mode wave energy decreases much as in the case of the wide impulse situation.

The spatio-temporal behaviour of the radial electric field component, $E_{\mathrm{x}}$, is given by the expression

$$
\begin{aligned}
E_{\mathrm{x}} & =q \pi^{3 / 2} \Theta\left(t-t_{0}\right) e^{-k_{\mathrm{y}}\left(x_{0}-x\right)} \\
& \cdot\left\{\sqrt{k_{\mathrm{y}}\left(x_{0}-x\right)}\left[F_{\mathrm{x}}(t)+F_{0}(t)\right]\right. \\
& \left.+\frac{i\left(t-t_{0}\right)}{\sqrt{k_{\mathrm{y}}\left(x_{0}-x\right)}} \frac{d \Omega(x)}{d x} F_{\mathrm{x}}(t)\right\}
\end{aligned}
$$
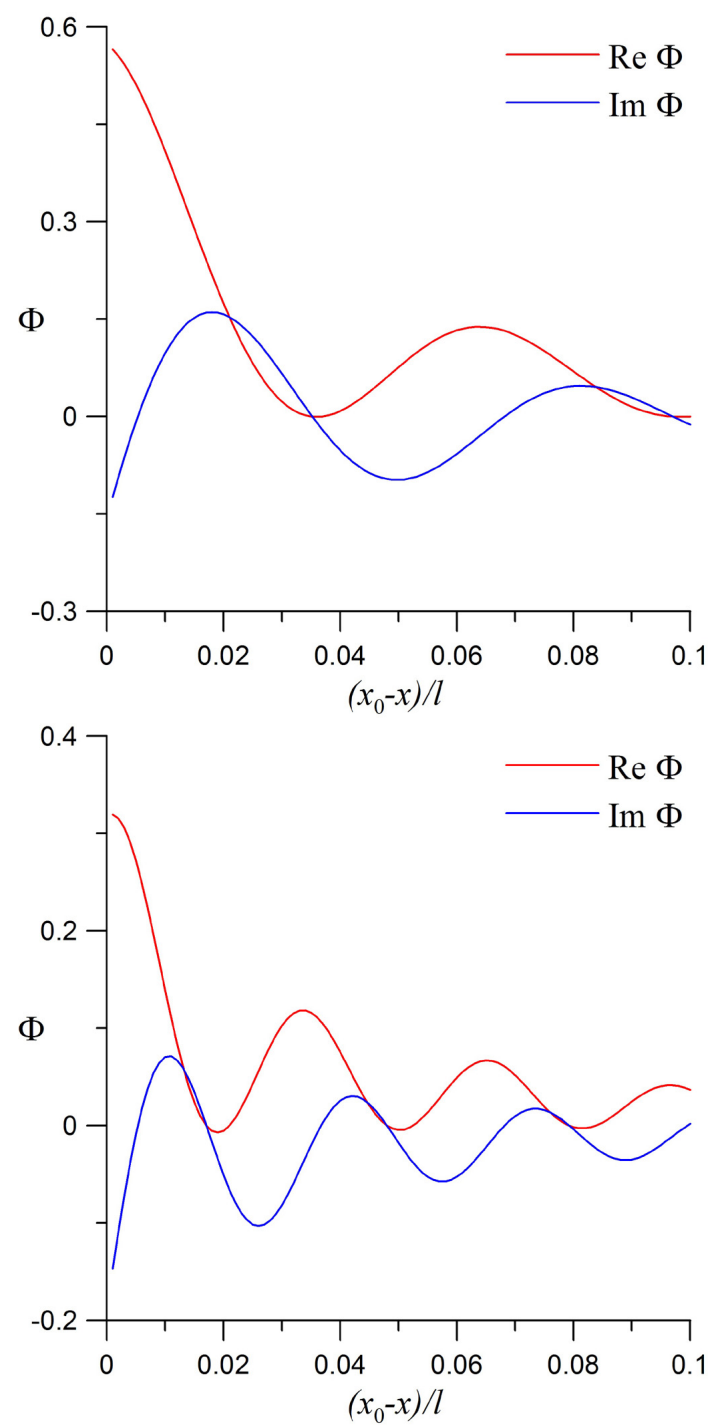

Fig. 2. Spatial structure of the wave at two times: $\Omega_{0}\left(t-t_{0}\right)=100$ (top panel) and $\Omega_{0}\left(t-t_{0}\right)=200$ (bottom panel).

(Fig. 5). There are two contributions in $E_{\mathrm{x}}$. The first is $F_{\mathrm{X}}(t)+F_{0}(t)$ which behaves similar to the $E_{\mathrm{y}}$ component: beating with decaying amplitude. The second one is proportional to $F_{\mathrm{X}}(t)$. It represents the oscillation with the local frequency $\Omega(x)$ on the given magnetic shell and grows with time as the square root of time. The amplitude of the $E_{\mathrm{X}}$ tends to some constant value, as is seen also from Fig. 4, where the temporal evolution of the toroidal mode electric field energy density $w_{\mathrm{x}}=\left|E_{\mathrm{X}}\right|^{2} / 8 \pi$ is shown. We conclude that the energy is transferred from the azimuthal component of the electric field to the radial component, or from poloidal field line oscillations into toroidal ones. This behaviour is similar to the wide impulse situation treated earlier by e.g. Hasegawa et al. (1983). However, contrary to that theory, the initial $E_{\mathrm{X}}$ value is not zero: at the outset, the amplitudes of $E_{\mathrm{x}}$ and $E_{\mathrm{y}}$ 


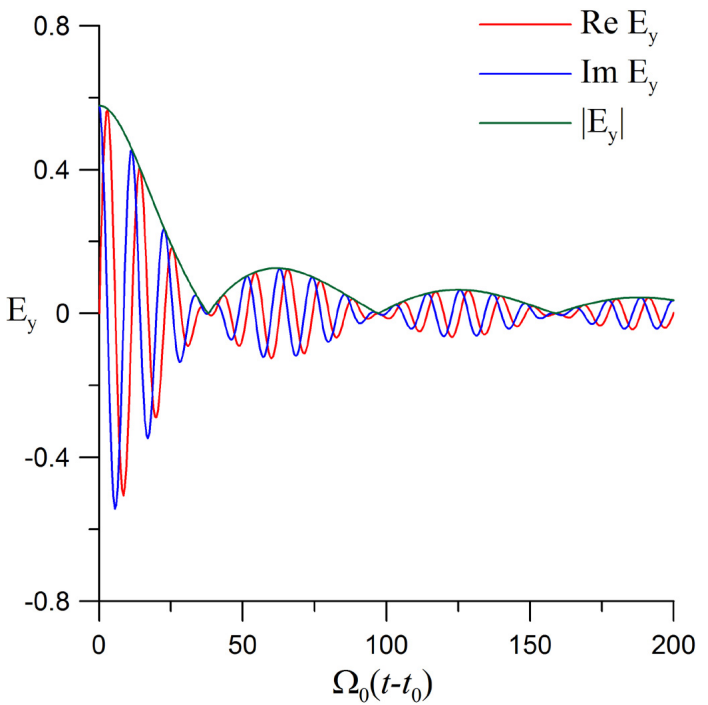

Fig. 3. Temporal evolution of $E_{\mathrm{y}}$ far from the source, $\left(x_{0}-x\right) / l=0.1$.

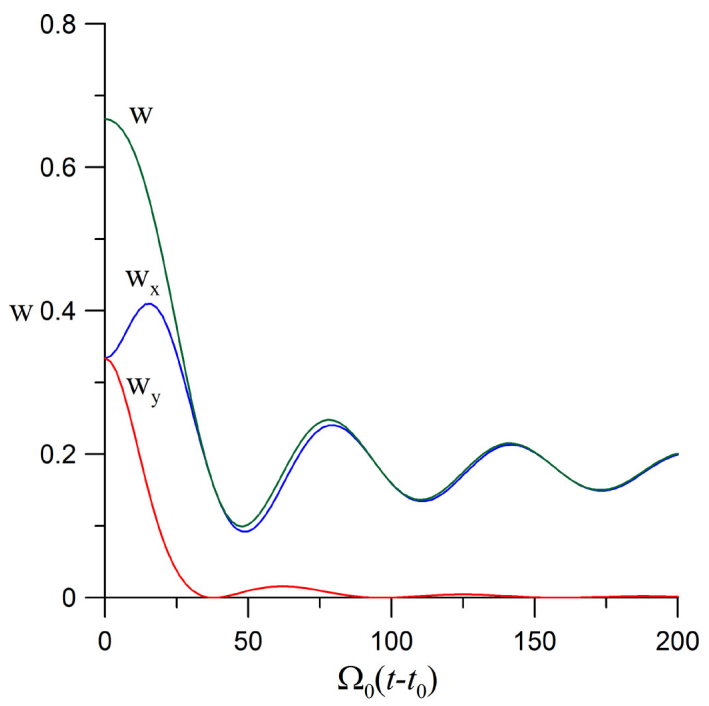

Fig. 4. Temporal evolution of wave energy density, $w$, and its components, $w_{\mathrm{x}}$ and $w_{\mathrm{y}}$, far from the source, $\left(x_{0}-x\right) / l=0.1$.

are equal to each other. This implies that the newborn wave exhibits a mixed polarization, poloidal and toroidal.

Another difference in comparison with the wide impulse theory is the total energy density, $w=w_{\mathrm{x}}+w_{\mathrm{y}}$ on the given magnetic shell is not constant (see Fig. 4). However, if the energy density decreases somewhere, it must grow somewhere else. Numerical calculations show that this happens on the surface of the impulse $x_{0}$; the energy increase is shown in Fig. 6. Because of this the total electric energy integrated over the whole plasma volume,

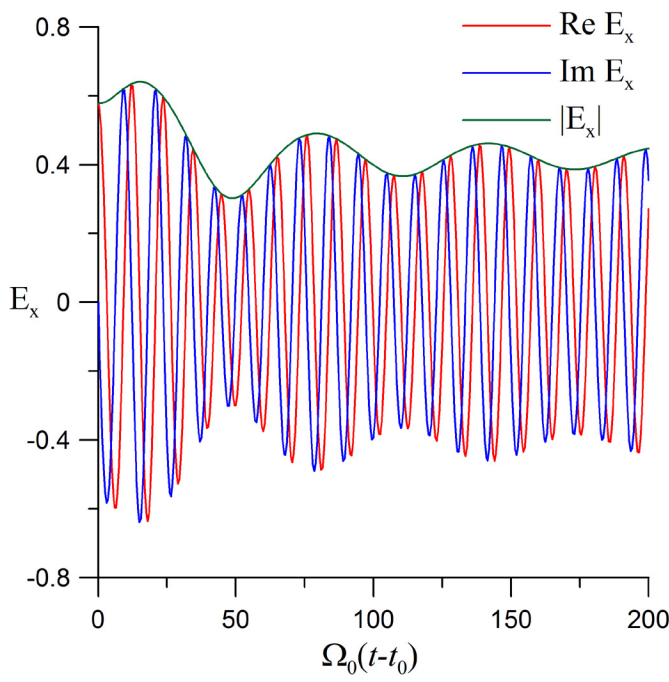

Fig. 5. Temporal evolution of $E_{\mathrm{X}}$ far from the source, $\left(x_{0}-x\right) / l=0.1$.

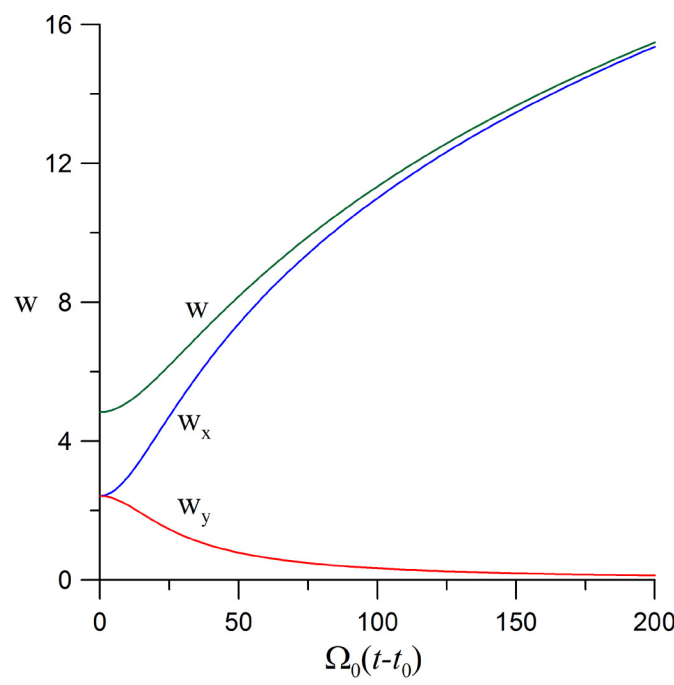

Fig. 6. Temporal evolution of wave energy density, $w$, and its components, $w_{\mathrm{X}}$ and $w_{\mathrm{y}}$, on the surface of the impulse $x_{0}$.

$$
W=\frac{1}{8 \pi} \int_{-\infty}^{\infty}\left(E_{\mathrm{x}} E_{\mathrm{x}}^{*}+E_{\mathrm{y}} E_{\mathrm{y}}^{*}\right) d x=\frac{1}{4} \pi^{3} q k_{\mathrm{y}},
$$

is conserved. To prove this one only needs to express $E_{\mathrm{X}}$ and $E_{\mathrm{y}}$ using Eqs. (13), (14), change an order of integration and use the integral representation of the delta-function.

Figure 7 displays the temporal change of the spatial wave structure represented by the wave energy density $w(x)$. At $t=t_{0}$, there is only a gradual decrease of the wave amplitude with distance from the source. As time elapses, the energy accumulates near the surface of the source, while far from the source it diminishes, tending to some small constant value. Thus, a kernel and a tail of the oscillation are formed. As is 


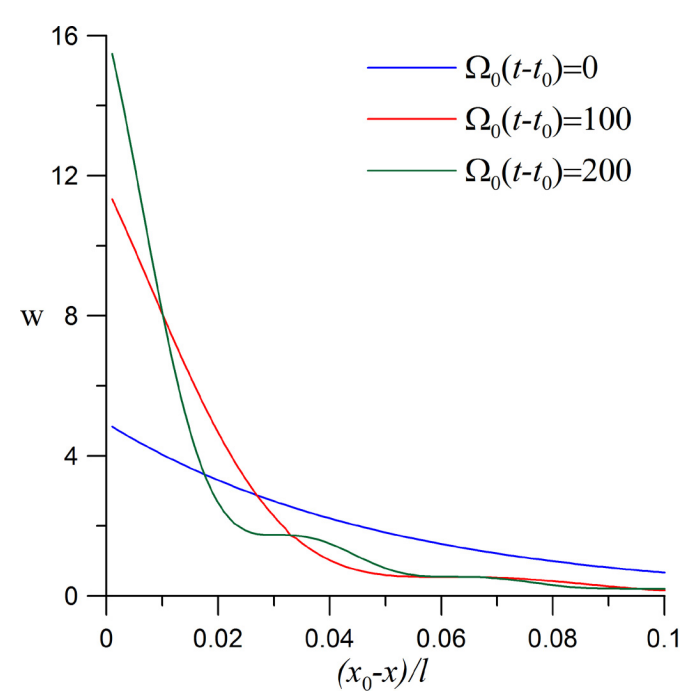

Fig. 7. Wave energy density, $w$, at different distances from the surface of the impulse $x_{0}$ for different times.

seen from the figure, the width of the kernel decreases with time.

\section{Conclusions}

This paper investigates the spatio-temporal structure of the high- $m$ ULF waves generated by a sudden impulse concentrated on a given magnetic shell in a rectangular box. Due to the assumed small spatial variation in the azimuthal coordinate, the contribution of the fast mode is negligibly small, and the wave is represented by the Alfvén mode. At the outset, both poloidal and toroidal components are present in the wave's magnetic field. The wave amplitude exponentially decreases with the distance from the source. The poloidal component oscillates in the beating regime. Due to phase mixing, its amplitude decreases with time down to zero. Beating is less pronounced in the toroidal component. Its amplitude grows with time, reaching some constant amplitude. The total energy, integrated over the entire plasma volume, is conserved. As time elapses, a thin kernel and a wide tail of oscillations are formed: the energy density near the source magnetic shell grows, while far from the source it is significantly decreasing, finally reaching some small constant value. That is, the Alfvén wave on the surface of the source with the local Alfvénic frequency on this surface grows. Each field line in the low amplitude tail oscillates with the local Alfvénic frequency on its magnetic shell.

The retreat of the wave energy onto the magnetic shell of the source is a rather peculiar finding. The initial poloidal wave spreads across field lines and excites local oscillations, decaying by phase mixing. The small radial scales generated in this way require a mode transformation towards toroidal oscillations. The amplitude of these oscillations decays with time, and energy conservation requires the wave energy to accumulate at the source magnetic field shell. The system behaves by not supporting wave energy transport in the radial direction in the long run. But this can only be achieved by coupling the initial poloidal wave energy into toroidal wave energy using the non-uniform background plasma and its wave dispersion properties as a kind of catalytical agent.

Many features of the solution derived here have analogies in the theory of the temporal evolution of an initial disturbance widely distributed across magnetic shells considered in several earlier publications. Among them are phase mixing, the transformation into toroidally polarized wave, and oscillations with the local Alfvénic frequency. These features were also described in the previous study of Hasegawa et al. (1983) dealing with wave generation by a surface impulse.

Other features, such as the beating on the poloidal component for a short time interval after the onset of the oscillations, the change of the mode polarization from a mixed to predominantly toroidal mode, the concentration of power near the surface of the source with the local Alfvénic frequency have no analogies in the theory of the initial disturbance widely distributed across the magnetic shells. These features were not revealed by the earlier study of Hasegawa et al. (1983). We suppose that such features can be used as observational marks of ULF waves generated by sudden impulses localized on some magnetic surface.

A factor which can prevent the concentration of power near the surface of the source is the dissipation of the wave energy due to the finite conductivity of the ionosphere. However, as is seen from Figs. 6 and 7, the power concentration becomes evident as soon as ten wave periods; since usual Pc4-5 pulsations are observable for the longer time, we do not expect the power concentration to be prevented by the dissipation. Second, our consideration is applied to the high$m$ waves, which are the subject of the various kinds of the wave-particle interactions and associated kinetic instabilities. These instabilities lead to the suppression of the dissipation on the earlier stages of the wave evolution (Klimushkin and Mager, 2004; Klimushkin, 2007).

The results of this paper can also be applied to the generation of magnetospheric perturbations due to buffeting of the magnetopause, especially traveling convection vortices (Kivelson and Southwood, 1991; Glassmeier, 1992). However, in this case the source is localized in both transverse coordinates, across L-shells and in the azimuthal direction. Furthermore, such sources should be moving in the azimuthal direction as they are related to magnetosheath flow, therefore the wave energy finally leaves the system. Mager and Klimushkin (2007, 2008), Zolotukhina et al. (2008) and Yeoman et al. (2012) considered ULF wave excitation by a moving source, but they supposed the source to be widely distributed across magnetic shells while localized in azimuth. A more general theoretical treatment is required here and will be the subject of future studies. The generation of the low- $m$ ULF waves by a surface impulse also demands a separate 
study, since the coupling with the fast mode greatly exaggerates a situation.

Acknowledgements. The work by D.K. and P.M. is supported by RFBR grants 12-02-00305-a and 12-05-98522-vostok-a, and by the Program of presidium of Russian Academy of Sciences \#22. D.K. was also supported by DAAD Research Fellowship program under grant A1104370. K.H.G. was financially supported by the German Ministry of Economy and Technology and the Deutsches Zentrum für Luft- und Raumfahrt under contract 50 OC 1001.

Topical Editor I. A. Daglis thanks P. Chi and W. J. Hughes for their help in evaluating this paper.

\section{References}

Agapitov, O., Glassmeier, K.-H., Plaschke, F., Auster, H.-U., Constantinescu, D., Angelopoulos, V., Magnes, W., Nakamura, R., Carlson, C. W., Frey, S., and McFadden, J. P.: Surface waves and field line resonances: A THEMIS case study, J. Geophys. Res., 114, A00C27, doi:10.1029/2008JA013553, 2009.

Akhiezer, A. I., Akhiezer, I. A., Polovin, R. V., Sitenko, A. G., and Stepanov, K. N.: Plasma electrodynamics. Vol. 1: Linear theory, International Series of Monographs in Natural Philosophy, Vol. 68, Pergamon Press, Oxford, UK, 1975.

Chen, L. and Hasegawa, A.: A theory of long period magnetic pulsation. 2. Impulse excitation of surface eigenmode, J. Geophys. Res., 79, 1033-1037, 1974.

Chi, P. J., Lee, D.-H., and Russell, C. T.: Tamao travel time of sudden impulses and its relationship to ionospheric convection vortices, J. Geophys. Res., 111, 1033-1037, doi:10.1029/2005JA011578, 2006.

DeKeyser, J., Roth, M., Reberac, F., Rezeau, L., and Belmont, G.: Resonant amplification of MHD waves in realistic subsolar magnetopause configurations, J. Geophys. Res., 104, 2399-2409, doi:10.1029/1998JA900060, 1999.

Fedorov, E., Mazur, N., Pilipenko, V., and Yumoto, K.: MHD wave conversion in plasma waveguides, J. Geophys. Res., 103, 2659526605, 1998.

Fujita, S., Glassmeier, K.-H., and Kamide, K.: MHD waves generated by the Kelvin-Helmholtz instability in a nonuniform magnetosphere, J. Geophys. Res., 101, 27317-27326, doi:10.1029/96JA02676, 1996.

Glassmeier, K.-H.: Traveling magnetospheric convection twinvortices - Observations and theory, Ann. Geophys., 10, 547-565, 1992.

Glassmeier, K.-H., Othmer, C., Cramm, R., Stellmacher, M., and Engebretson, M.: Magnetospheric field line resonance: a comparative approach, Surveys Geophys., 20, 61-109, 1999.

Hasegawa, A. and Chen, L.: Theory of magnetic pulsations, Space Sci. Rev., 16, 347-359, 1974.

Hasegawa, A. and Uberoi, C.: The Alfvén wave, US Dept. of Energy, Washington, 1982.

Hasegawa, A., Tsui, K. N., and Assis, A. S.: A theory of long period magnetic pulsations. 3. Local field line oscillations, Geophys. Res. Lett., 10, 765-767, 1983.

Kivelson, M. G. and Southwood, D. J.: Ionospheric traveling vortex generation by solar wind buffeting of the magnetosphere, J. Geophys. Res., 96, 1661-1667, doi:10.1029/90JA01805, 1991.
Klimushkin, D. Y.: Method of description of the Alfvén and magnetosonic branches of inhomogeneous plasma oscillations, Plasma Phys. Rep., 20, 280-286, 1994.

Klimushkin, D. Y.: How energetic particles construct and destroy poloidal high- $m$ Alfvén waves in the magnetosphere, Planet. Space Sci., 55, 722-730, doi:10.1016/j.pss.2005.11.006, 2007.

Klimushkin, D. Yu. and Mager, P. N.: The spatio-temporal structure of impulse-generated azimuthalsmall-scale Alfvén waves interacting with high-energy chargedparticles in the magnetosphere, Ann. Geophys., 22, 1053-1060, doi:10.5194/angeo-221053-2004, 2004.

Klimushkin, D. Y., Podshibyakin, I. Y., and Cao, J. B.: Azimuthally small-scale Alfvén waves in magnetosphere excited by the source of finite duration, Earth, Planets, Space, 59, 951-959, 2007.

Leonovich, A. S. and Mazur, V. A.: Standing Alfvén waves in an axisymmetric magnetosphere excited by a non-stationary source, Ann. Geophys., 16, 914-920, doi:10.1007/s00585-998-0914-z, 1998.

Leonovich, A. S. and Mazur, V. A.: Standing Alfvén waves in the magnetosphere from a localized monochromatic source, J. Geophys. Res., 104, 2411-2420, 1999.

Mager, P. N. and Klimushkin, D. Y.: Theory of azimuthally smallscale Alfvén waves in an axisymmetric magnetosphere with small but finite plasma pressure, J. Geophys. Res., 107, A11, doi:10.1029/2001JA009137, 2002.

Mager, P. N. and Klimushkin, D. Y.: Generation of Alfvén waves by a plasma inhomogeneity moving in the Earth's Magnetosphere, Plasma Phys. Rep., 33, 391-398, doi:10.1134/S1063780X07050042, 2007.

Mager, P. N. and Klimushkin, D. Yu.: Alfvén ship waves: high$m$ ULF pulsations in the magnetosphere generated by a moving plasma inhomogeneity, Ann. Geophys., 26, 1653-1663, doi:10.5194/angeo-26-1653-2008, 2008.

Mann, I. R. and Wright, A. N.: Finite lifetimes of ideal poloidal Alfvén waves, J. Geophys. Res., 100, 23677-23686, 1995.

Mazur, V. A. and Chuiko, D. A.: Excitation of a magnetospheric MHD cavity by Kelvin-Helmholtz instability, Plasma Phys. Rep., 37, 913-934, doi:10.1134/S1063780X11090121, 2011.

Mishin, V. V.: Accelerated motions of the magnetopause as a trigger of the Kelvin-Helmholtz instability, J. Geophys. Res., 98, 2136521371, doi:10.1029/93JA00417, 1993.

Morse, P. M. and Feshbach, H.: Methods of Theoretical Physics, Part I, McGraw-Hill, New York, 1953.

Plaschke, F. and Glassmeier, K.-H.: Properties of standing KruskalSchwarzschild-modes at the magnetopause, Ann. Geophys., 29, 1793-1807, doi:10.5194/angeo-29-1793-2011, 2011.

Radoski, H. R.: A theory of latitude dependent geomagnetic micropulsations: the asymptotic fields, J. Geophys. Res., 79, 595613, 1974.

Yeoman, T. K., M., J., Mager, P. N., and Klimushkin, D. Y.: SuperDARN observations of high- $m$ ULF waves with curved phase fronts and their interpretation in terms of transverse resonator theory, J. Geophys. Res., 117, A06231, doi:10.1029/2012JA017668, 2012.

Zolotukhina, N. A., Mager, P. N., and Klimushkin, D. Yu.: Pc5 waves generated by substorm injection: a case study, Ann. Geophys., 26, 2053-2059, doi:10.5194/angeo-26-2053-2008, 2008. 